\title{
Psychische Versorgung von Kindern und Jugendlichen in Österreich neu aufstellen: Dringender Handlungsbedarf besteht JETZT !
}

\author{
Paul L. Plener · Claudia M. Klier · Leonhard Thun-Hohenstein · Kathrin Sevecke
}

Angenommen: 10. November 2021

(c) The Author(s), under exclusive licence to Springer-Verlag GmbH Austria, ein Teil von Springer Nature 2021

\section{Internationale Lage}

Im Zuge der aktuellen CoVid-19 Pandemie zeigt sich weltweit wie auch in Österreich eine deutliche Zunahme an psychischen Erkrankungen mit gestiegenen Raten an Depressionen, Angststörungen, Essstörungen, Suizidgedanken sowie Suizidversuchen [2, 4, 6]. Dabei handelt es sich um kein nur in Österreich existierendes Phänomen, entsprechend gibt es ähnliche Signale aus anderen Nationen [7, 8, 10]. So hat auch die amerikanische kinder- und jugendpsychiatrische Fachgesellschaft (AACAP) zuletzt gemeinsam mit der amerikanischen Fachgesellschaft für Kinder- und Jugendheilkunde (AAP) auf nationalen Notstand im Bereich der seelischen Kinder- und Jugendgesundheit hingewiesen [1]. Das National Health Service (NHS) in Großbritannien hat seine Ausgaben im Bereich der

Univ. Prof. Dr. P. L. Plener, MHBA ( $ه)$

Universitätsklinik für Kinder- und Jugendpsychiatrie, Medizinische Universität Wien, Währingergürtel 18-20, 1090 Wien, Österreich

Klinik für Kinder- und Jugendpsychiatrie und Psychotherapie, Universität Ulm, Ulm, Deutschland paul.plener@meduniwien.ac.at

\section{M. Klier}

Universitätsklinik für Kinder- und Jugendheilkunde, Medizinische Universität Wien, Wien, Österreich

Comprehensive Center for Pediatrics, Medizinische Universität Wien, Wien, Österreich

L. Thun-Hohenstein

Universitätsklinik für Kinder- und Jugendpsychiatrie, Paracelsus Medizinische Privatuniversität Salzburg, Salzburg, Österreich

\section{K. Sevecke}

Universitätsklinik für Psychiatrie, Psychotherapie und Psychosomatik im Kindes- und Jugendalter, Medizinische Universität Innsbruck, Innsbruck, Österreich
Versorgung psychischer Erkrankungen bei Kinder und Jugendlichen massiv erhöht und einen Forderungskatalog für die bessere Versorgung psychisch erkrankter Kinder und Jugendlicher präsentiert [5]. Zuletzt hat auch die UNICEF auf die sich dramatisch zuspitzende Lage im Bereich der Versorgung psychisch kranker Kinder und Jugendliche hingewiesen und in diesem Zusammenhang auch die massiven Folgekosten einer Nicht-Behandlung psychischer Erkrankung im Lebensalter der 0-18-jährigen thematisiert [9].

\section{Österreichische Situation}

Die österreichische Versorgungslandschaft für die Betreuung psychisch erkrankter Kinder und Jugendlicher war bereits vor der Pandemie unzureichend ausgestattet. Es besteht ein durchgängiger Mangel an kassenfinanzierter Psychotherapie für Kinder- und Jugendliche, der Ausbau niederschwelliger Angebote an Schulen ist im Vergleich $\mathrm{zu}$ vielen anderen europäischen Ländern nie erfolgt. Es existiert kein flächendeckendes Konzept zur Prävention psychischer Erkrankungen. Die kinder- und jugendpsychiatrische Versorgung hat noch nie die im Strukturplan Gesundheit Österreich geforderten Versorgungsziele, weder im ambulanten, noch im stationären Bereich erreicht [3]. Das führt dazu, dass weniger als die Hälfte $(47,5 \%)$ jener Kinder und Jugendlicher, die über eine psychische Erkrankung in ihrer Lebenszeit berichten, irgendeine Form der Behandlung erhalten haben [11]. Auch wenn vielerorts bemerkenswerte Modellprojekte existieren, so muss doch festgehalten werden, dass die Versorgung psychisch kranker Kinder- und Jugendliche in Österreich bereits vor der Pandemie unzureichend gegeben war. Durch die nun gestiegenen Zahlen psychischer Erkrankungen ist das System mittlerweile am Rande seiner Kapazitäten angekommen, bzw. weit darüber hinaus belastet und 
wird derzeit nur durch die persönliche Bereitschaft der in diesem System Arbeitenden über die Belastungsgrenzen hinaus zu arbeiten, aufrechterhalten.

In den letzten Monaten konnten erste wichtige Schritte erzielt werden, es gab erste zusätzliche $\mathrm{Zu}$ wendungsversprechen seitens des Bundesministeriums für Soziales, Gesundheit, Pflege und Konsumentenschutz, ebenso wurde die Kinderchancen Initiative seitens des Bundesministerium präsentiert, die eine Neuausrichtung, basierend auf EU Geldern vorantreiben soll. Aus unserer Sicht sind das begrüßenswerte erste Schritte, es wird jedoch ein Vielfaches der bislang veranschlagten Mittel und ein „Neu-Denken“ der Versorgungsstruktur brauchen, um den nun gestiegenen Anforderungen gerecht zu werden.

Vor diesem Hintergrund fordern wir als Österreichische Gesellschaft für Kinder- und Jugendpsychiatrie, Psychosomatik und Psychotherapeutische Medizin ein klares Bekenntnis der Politik sowohl auf Bundes- sowie auch auf Länderebene die Finanzierung im Bereich der Versorgung psychischer Erkrankungen bei Kindern- und Jugendlichen erheblich zu steigern und gesetzliche Rahmenbedingungen zu schaffen, um die Versorgung zu verbessern und zu flexibilisieren.

Dabei wird es neben dem zur Verfügung stellen von frei zugänglichen, kassenfinanzierten Leistungen auch darum gehen das Versorgungssystem so neu zu denken, dass ein flächendeckender, niederschwelliger Zugang gewährleistet werden kann.

\section{Forderungen der ÖGKJP}

In diesem Zusammenhang fordern wir:

- Zugang zu kassenfinanzierter Psychotherapie und kinder- und jugendpsychiatrischen Angeboten.

Alle diesbezüglichen Leistungen müssen für alle Kinder- und Jugendliche egal in welchem Umfeld sie aufwachsen, kostenfrei erhältlich sein. Hier müssen Rahmenbedingungen im Sinne von Qualifizierungsnachweisen geschaffen werden, sodass eine psychotherapeutische Behandlung von Kindern und Jugendlichen ausschließlich durch Personen erbracht wird, die in diesem Bereich nachweislich eine spezifische Qualifikation erworben haben

- Stellenausbau im Bereich der Kinder- und Jugendpsychiatrie, der Kinder- und Jugendhilfe sowie im Bereich der Kinder- und Jugendlichen Psychotherapie.

In diesem Zusammenhang müssen auch gesetzlich neue Modelle geschaffen werden um die Ausbildungskapazität im Mangelfach Kinder- und Jugendpsychiatrie und Psychotherapeutische Medizin zu erhöhen um hier längerfristig mehr Fachärztinnen und Fachärzte auf diesem Gebiet auszubilden.

- Umgestaltung der Angebote mit vermehrter Verankerung niedrigschwelliger therapeutischer Angebote im Alltag von Kindern und Jugendlichen.
Unter Einbezug eines vermehrten Angebotes an Schulsozialarbeit, Schulpsychologie und einer Koordination mit dem schulärztlichen System, gegebenenfalls auch unter Einbezug von neu zu schaffenden „Mental Health Nurses“ soll eine weitere Stufe in einem gestuften Versorgungssystem entstehen, die im Lebenskontext von Kindern und Jugendlichen vorhanden ist. Ähnliche Modelle müssen auch für den Bereich der Ausbildungen von Lehrlingen geschaffen werden. Es wird hier darum gehen Versorgungsketten darzustellen, so dass diese niederschwelligen Bereiche optimal mit den Bereichen der Psychotherapie und der ambulanten und stationären Kinder- und Jugendpsychiatrie verknüpft werden. In dem Zusammenhang müssen auch stepped care Modelle (sowohl für das Stepping Up sowie das Stepping Down) entwickelt werden.

- Schaffung und Ausbau von digitalisierten Angeboten.

Auch diese sollen ein niederschwelliges Angebot darstellen und in stepped care Angebote integriert werden. Hierzu bedarf es dringend der Sichtung von Angeboten von bereits existierenden Tools und der Schaffung neuer Tools in noch nicht abgedeckten Bereichen durch Forschung. Es bedarf eines Qualitäts- und Wirksamkeitsnachweises um dann auch von der öffentlichen Hand zur Verfügung gestellt werden zu können.

- Investition in Frühintervention:

Wie die UNICEF [9] aktuell deutlich herausstellt, ist eine Investition in frühe Maßnahmen hinsichtlich des Nutzen für die seelische Gesundheit (und nicht zuletzt auch hinsichtlich der Rentabilität in einem volkswirtschaftlichen Kontext) die beste Investition (im Sinne eines „Return on Investment“). Weitere finanzielle Stützung des Bereichs frühe Hilfen, ebenso wie zusätzliche Unterstützung und Vernetzung im Bereich Elementarpädagogik mit kinderund jugendtherapeutischen und kinder- und jugendpsychiatrischen Angeboten ist hier zu fordern.

- Systemübergreifende Kooperation:

Die Finanzierung von Kooperationen im Sinne einer Vernetzung zwischen Kinder- und Jugendhilfe und Kinder- und Jugendpsychiatrie muss sichergestellt werden. Solche Kooperationsmodelle beruhen derzeit auf reiner Freiwilligkeit und werden aus den Strukturen heraus getragen. Hier bedarf es einer deutlich besseren Vernetzung, die für solch eine Arbeit auch entsprechend honoriert werden muss.

- Flächendeckende Einführung evidenzbasierter Präventionsprogramme im schulischen sowie im außerschulischen Rahmen:

Während bereits jetzt Präventionsprogramme, deren Effektivität eindrücklich nachgewiesen wurde, existieren, so harren diese Programme weiterhin einer flächendeckenden Umsetzung, was vor allem eine Kooperation zwischen dem Bildungs- und dem Gesundheitsbereich notwendig machen würden. Vor allem im Bereich der Suizidprävention wie auch 
im Bereich der Mobbingprävention sind zusätzliche Mittel zur Verfügung zu stellen.

- Förderung der „Mental Health Literacy“ bei Kindern im Bereich der Elementarpädagogik aber auch im schulischen- und im Ausbildungsbereich.

Hierfür müssen Lehrkonzepte entwickelt werden, so dass das Identifizieren von seelischen Erkrankungen Kindern und Jugendlichen leichter möglich ist und diese auch über Möglichkeiten zur Hilfe informiert werden.

- Regelmäßiges Monitoring der psychischen Gesundheit von Kindern und Jugendlichen in Österreich. In Österreich gibt es derzeit kein regelmäßig in Wellen durchgeführtes Monitoring von psychischer Gesundheit, wie es etwa in vielen anderen Nationen (Deutschland, USA, Großbritannien) durchgeführt wird. Hier ist zu fordern, dass zumindest 2-jährig eine repräsentative Erhebung zur psychischen Gesundheit von Kindern und Jugendlichen erfolgt, um frühzeitig Trends zu erkennen und gegensteuern zu können.

Interessenkonflikt P.L. Plener has received research funding from the German Federal Ministry of Education and Research (BMBF), the German Federal Institute of Drugs and Medical Devives (BfARM), Baden Wuerttemberg Foundation, Volkswagen Foundation, Vienna Landeszielsteuerungskommission. Lundbeck and Servier. He received an advisorer's honorarium from Boehringer-Ingelheim and speaker honoraria from Shire, Janssen Neuroscience, Infetopharm, Gerrot Lannach and Oral B. C.M. Klier has the following conflicts of interests: Speaker Sandoz 2020, Cooperation with Trigal Pharma without any financial involvement since 2018PI and country coordinator League study Takeda/Shire SPD 503-401. L. ThunHohenstein und K. Sevecke geben an, dass kein Interessenkonflikt besteht.

\section{Literatur}

1. AACAP (2021) A declaration from the American Academy of Pediatrics, American Academy of Child and Adolescent Psychiatry and Children's Hospital Association. Download unter: https://www.aap.org/en/advocacy/child-andadolescent-healthy-mental-development/aap-aacapcha-declaration-of-a-national-emergency-in-child-andadolescent-mental-health/.Zugegriffen: 29.10.2021.

2. Dale R, O'Rourke T, Humer E, Jesser A, Plener PL, Pieh C. Mental health of apprentices during the COVID-19 pandemic in Austria and the effect of gender, migration back- ground, and work situation. Int J Environ Res Publ Health. 2021;18:8933.

3. FliedlR, Ecker B, KarwautzA. Kinder-undjugendpsychiatrischeVersorgung 2019 in Österreich-Stufen der Versorgung, Ist-Stand und Ausblick. Neuropsychiatrie. 2020;34:179-88.

4. Humer E, Dale R, Plener PL, Probst T, Pieh C. Mental health of high school students one semester after lifting of COVID19-related remote schooling measures in Austria. Jama Netw Open. 2021. In press.

5. NHS. NHS (2021) Reaching the tipping point: Children and young people's mental health. Download unter.. https://www.nhsconfed.org/publications/reachingtipping-point.Zugegriffen: 29.10.2021.

6. Pieh C, Plener PL, Probst T, Dale R, Humer E. Assessment of Mental Health of High School Students During Social Distancing and Remote Schooling During the COVID-19 Pandemic in Austria. Jama Netw Open. 2021;4(6):e2114866. https://doi.org/10.1001/jamanetworkopen.2021.14866.

7. Ravens-Sieberer U, Kaman A, Erhart M, Otto C, Devine J, Löffler C, Hurrelmann K, Bullinger M, Barkmann C, SiegelNA, Simon AM, Wieler LH, SchlackR, Hölling H (2021) Quality oflifeand mentalhealthin children and adolescents during the first year of the COVID-19 pandemic: results of a two-wave nationwide population-based study. Eur Child Adolesc Psychiatry. 2021 Oct 12:1-14. https://doi.org/10. 1007/s00787-021-01889-1. Onlineim Druck.

8. Ravens-Sieberer U, Kaman A, Otto C, Adedeji A, Devine J, Erhart M, Napp AK, Becker M, Blanck-Stellmacher U, Löffler C, Schlack R, Hurrelmann K (2020) Mental health and quality of life in children and adolescents during the COVID-19 pandemic - results of the COPSY study. Dtsch Arztebl Int 117:828-829.

9. UNICEF (2021) The State of the World's Children 2021: On My Mind: Promoting, protecting and caring for children's mental health. Download unter: https://www. unicef.org/reports/state-worlds-children-2021. Zugegriffen:29.10.2021.

10. Vizard T, Sadler K, Ford T, Newlove-Delgado T, McManus S, Marcheselli F, Davis J, Williams T, Leach C, Mandalia D, Cartwright C (2020) Mental Health of Children and Young People in England, 2020: Wave 1 follow up to the 2017 survey. Download unter: https://digital.nhs.uk/data-andinformation/publications/statistical/mental-health-ofchildren-and-young-people-in-england/2020-wave-1follow-up\#chapter-index. Zugegriffen: 29.10.2021.

11. Wagner G, Zeiler M, Waldherr K, Philipp J, Truttmann S, Dür W, et al. Mental health problems in Austrian adolescents: a nationwide, two-stage epidemiological study applying DSM-5 criteria. Eur Child Adolesc Psychiatry. 2017;26(12):1483-99.

Hinweis des Verlags Der Verlag bleibt in Hinblick auf geografische Zuordnungen und Gebietsbezeichnungen in veröffentlichten Karten und Institutsadressen neutral. 